
3 Research Square
Preprints are preliminary reports that have not undergone peer review.
They should not be considered conclusive, used to inform clinical practice,
or referenced by the media as validated information.

\title{
Urban-rural differential in Hypertension and Diabetes among Elderly in India: A study of prevalence, factors, and treatment-seeking
}

Shekhar Chauhan

International Institute for Population Sciences

Ratna Patel

International Institute for Population Sciences

Samriddhi S Gupte

Max Institute of Healthcare Management, Indian School of Business

Shubham Kumar ( $\square$ shubhamk98@gmail.com )

International Institute for Population Sciences

\section{Research Article}

Keywords: Hypertension, Diabetes, Urban-rural differential, Treatment-seeking, India

Posted Date: May 4th, 2021

DOI: https://doi.org/10.21203/rs.3.rs-488657/v1

License: (c) (i) This work is licensed under a Creative Commons Attribution 4.0 International License. Read Full License

Version of Record: A version of this preprint was published at Diabetes \& Metabolic Syndrome: Clinical Research \& Reviews on July 1st, 2021. See the published version at https://doi.org/10.1016/j.dsx.2021.102201. 


\section{Abstract}

Objectives: The study estimates the prevalence of hypertension and diabetes among older adults and bring forth the urban-rural differentials in the said morbidities. The treatment-seeking approach of older adults is also looked at with regard to hypertension and diabetes.

Methods: The data for this study come from the Longitudinal Ageing Study in India (LASI) conducted in 2017-18. Bivariate analyses were used to understand the rural-urban gap in hypertension and diabetes with socioeconomic and demographic parameters. Further, logistic regression was used to check the likelihood of hypertension and diabetes with socioeconomic and demographic variables. Finally, a non-linear decomposition technique, Fairlie's decomposition technique was applied to check the difference in the probability of hypertension and diabetes between rural and urban by estimating contributions of a group (rural-urban) differences.

Results: Study noted a higher prevalence of hypertension and diabetes among elderly residing in urban areas than their counterparts. Prevalence of hypertension and diabetes was higher among those aged 70+, elderly females, less educated, and non-poor. Education status alone accounts for more than four-fifths $(88.62 \%)$ and more than half $(52.02 \%)$ of the inequality in the prevalence of urban-rural hypertension and diabetes, respectively. Elderly with higher education were 2.88 times $(\mathrm{OR}=2.88 ; \mathrm{C} . \mathrm{I} .=1.40-5.90)$ more likely to sought treatment for hypertension than uneducated older people in urban areas

Conclusion: Since treatment-seeking is relatively low among elderly in poor households, practices must be identified for a poverty-stricken elderly population to overcome the financial barriers that may prevent the elderly from seeking and complying with treatment.

\section{Introduction}

Globally, non-communicable diseases (NCDs) cause the death of nearly 41 million people every year, equivalent to $71 \%$ of the total deaths (GBD 2015). Cardiovascular diseases account for most NCD deaths (17.9 million), followed by cancer, respiratory diseases, and diabetes (1.5 million) [1]. Hypertension is routinely associated with developing various cardiovascular diseases such as ischemic heart disease, heart failure, strokes, etc. [2-3]. The Global Burden of Disease (2015) listed hypertension as one of the top causes of not only mortality but also for loss of disability-adjusted life years [1]. Similarly, diabetes is also a risk factor for cardiovascular disease. Both hypertension and diabetes are often prevalent among older adults as both these diseases are directly proportional to age [4]. India's hypertension and diabetes story look grim in itself. India serves as the diabetes capital for the world, with every fifth diabetic person in the world happens to be an Indian [5]. A meta-analysis conducted on hypertension points towards its overall prevalence being $29.8 \%$ in India, with several studies showing an alarming increase in the prevalence rates [6].

Within India, there exist regional differences in the prevalence of hypertension and diabetes. These regional differences in hypertension range from 14.5$35.8 \%$ across India [6] and for diabetes, ranging from 3.8-21\% [7]. There is also a stark urban-rural divide in the prevalence of both diseases. Various studies show that urban areas are in greater distress than their rural counterparts [6-9]. These variations can be attributed to differences in geographic locations and socio-economic status, lifestyle, dietary habits, etc. [9]. These socio-economic variables are responsible for disparity in prevalence of chronic diseases and variation in the treatment-seeking behavior for the diseases. Health-care seeking behavior is believed to be the summation of environmental factors (such as areas living in) and individual characteristics [10]. A study shows that out of their sample, one-third of patients were aware of their condition; out of these, twothird were on treatment [11]. The education level of an individual plays a significant role in their awareness and facilitates accepting the diagnosis and adapting to the behavioral changes required [12].

As the world steps towards an increase in the aged population, it is safe to assume that morbidities such as hypertension and diabetes would take precedence over any other in terms of its prevalence. With the growing elderly population in India, the burden of these morbidities is bound to increase. Hence, there is an urgent need to understand and factor in the urban-rural divide in the prevalence of hypertension and diabetes to have a targeted approach to tackle the problem. Simultaneously it is also of utmost importance to look into the treatment-seeking approach of patients with hypertension and diabetes to identify the bottlenecks and ensure accessibility and willingness to use formal healthcare services.

The objective of the present study is to estimate the prevalence of hypertension and diabetes among older adults and bring forth the urban-rural differentials in the said morbidities. The treatment-seeking approach of older adults is also looked at with regard to hypertension and diabetes.

\section{Material And Methods}

\subsection{Data:}

The data for this study come from the Longitudinal ageing study of India (LASI) wave one conducted in 2017-18. LASI is the first-ever dataset in India that provides longitudinal data for designing policies and programs in the broad domain of the older population's social, health, and economic well-being. The study was conducted in all 35 states and union territories (except Sikkim). LASI included Indian adults and older adults and women age 45 years and above.

The study adopted a multistage stratified area probability cluster sampling design to collect information from 45 years older adults and above and their spouses irrespective of age. The broader information included chronic diseases among older adults and the elderly population across the socioeconomic spectrum in India and its states and territories. The health modules questions had overall health and specific chronic diseases, organ-based chronic health conditions, and symptoms-based health conditions. Health professionals diagnose chronic diseases like hypertension and diabetes.

The study included a total of 72,250 individuals age 45 years and above, including 31,464 elderly aged 60 years and above and 6,749 oldest persons aged 75 years and above. There was no upper age limit for sample selection, including the collection of biomarkers. 


\subsection{Outcome variables:}

The dependent variables for this study are hyphenation and diabetes. These health outcomes are based on diagnosis by health professionals. The question included, "has any health professional diagnose you with hypertension and diabetes?" Another outcome variable for this study is the treatment behaviour which was asked, "In order to control your blood pressure or hypertension/ blood sugar or diabetes, are you currently taking any medication?"

\subsection{Predictor variables:}

The study includes some selected socioeconomic and demographic variables which explain the rural-urban gap in hypertension and diabetes among older. The independent variables are age (60-69 years; 70+), marital status (Currently married; never married; divorced/separated/deserted), education (No education; below primary; primary; secondary; higher), religion (Hindu; Muslim; others), currently working (Yes; No), ever used smokeless tobacco (Yes; No) and ever consumed alcohol (Yes; No).

\subsection{Statistical analysis:}

To estimate the prevalence of hypertension and diabetes in rural and urban, bivariate analyses were used to understand the rural-urban gap in hypertension and diabetes with socioeconomic and demographic parameters. Further, logistic regression was used to check the likelihood of hypertension and diabetes with socioeconomic and demographic variables in rural and urban areas. The result was present in the form of an odds ratio, with $95 \%$ confidence intervals. Finally, a non-linear decomposition technique like Fairlie's decomposition technique was applied to check the difference in the probability of hypertension and diabetes between rural and urban by estimating contributions of a group (rural-urban) differences. Hence, the non-linear decomposition function, $Y=F\left(X_{i} \beta\right)$, can be express as,

$$
Y^{U}-Y^{R}=\left[\sum_{i=1}^{N^{U}} \frac{F\left(x_{i}^{U} \beta^{U}\right)}{N^{U}}-\sum_{i=1}^{N^{R}} \frac{F\left(x_{i}^{R} \beta^{U}\right)}{N^{R}}\right]+\left[\sum_{i=1}^{N^{R}} \frac{F\left(x_{i}^{R} \beta^{U}\right)}{N^{R}}-\sum_{i=1}^{N^{R}} \frac{F\left(x_{i}^{R} \beta^{R}\right)}{N^{R}}\right]
$$

Where, $\mathrm{N}^{\mathrm{j}}$ is the sample size for group $\mathrm{j}\left(\mathrm{j}=\mathrm{U}\right.$ \& $\mathrm{R}$ Urban and Rural), $\mathrm{Y}^{\wedge} \mathrm{j}$ is the average probability of a binary outcome of interest for group $\mathrm{j}, \mathrm{F}$ is the cumulative distribution function from the logistic distribution, $x_{i}$ is the set of the average value of the independent variable in group $j$, and $\beta$ is the coefficient estimates for the group $\mathrm{j}$. the first term in brackets represents the part of the urban-rural gap that is due to group differences in distributions of $x_{i}$. The second term means the part due to differences in the coefficient to the exogenous covariates.

\section{Results}

Table 1 depicts the result for socio-demographic characteristics of the elderly along with the prevalence of hypertension and diabetes among them by various background characteristics. The result noted a higher prevalence of hypertension and diabetes among elderly residing in urban areas than their counterparts for each background characteristic. Furthermore, it was noted that the prevalence of hypertension and diabetes was higher among those aged $70+$, females, divorced/separated/deserted, less educated, and non-poor.

Table 2 estimated the odds ratio for hypertension and diabetes among the elderly by their socio-demographic characteristics, separately for rural and urban areas. Results found that females residing in rural areas were 1.47 times more likely to be diagnosed with hypertension than their male counterparts residing in rural areas. Similarly, females residing in urban areas were 1.43 times more likely to be diagnosed with hypertension than their male counterparts residing in urban areas. Never-married elderly were less likely to be diagnosed with hypertension $(O R=0.45 ; C . I .=0.21-0.95)$ and diabetes $(O R=0.20 ; C . I .=0.06-0.65)$ than older people who were currently married in rural areas.

Education among the elderly depicts that those who attained higher education were more likely to be diagnosed with hypertension $(\mathrm{OR}=2.09 ; \mathrm{C} . \mathrm{I} .=1.50-2.91)$ and diabetes $(\mathrm{OR}=3.16 ; \mathrm{C} . \mathrm{I} .=2.01-4.96)$ than those who were uneducated in rural areas. Similarly, elderly with higher education were 2.12 times $(\mathrm{OR}=2.12$; C.I. $=1.40-3.21)$ more likely to be diagnosed with hypertension and 1.89 times $(O R=1.89 ; C . I .=1.20-2.97)$ more likely to be diagnosed with diabetes in urban areas than those elderly who were uneducated. Non-poor older people were 1.54 times $(\mathrm{OR}=1.54 ; \mathrm{C} . \mathrm{I} .=1.37-1.73)$ more likely to be diagnosed with hypertension and diabetes $(\mathrm{OR}=1.54$; C.I. $=1.30-1.83)$ than poor people in rural areas, also, non-poor people were more likely to diagnosed with diabetes $(\mathrm{OR}$ $=1.53 ;$ C.I. $=1.23-1.89)$ than poor people in urban areas. 
Table 1

Sociodemographic characteristics and prevalence of hypertension and diabetes among elderly in urban and rural areas

\begin{tabular}{|c|c|c|c|c|c|c|c|c|c|c|}
\hline & & Rural & & & Urban & & & Total & & \\
\hline & & Hypertension & Diabetes & $\mathrm{N}$ & Hypertension & Diabetes & $\mathrm{N}$ & Hypertension & Diabetes & $\mathrm{N}$ \\
\hline \multirow[t]{2}{*}{ Age group } & $60-69$ & 25.7 & 9.5 & 12,120 & 44.5 & 26.5 & 6,321 & 31.2 & 14.5 & 18 \\
\hline & $70+$ & 28.6 & 9.1 & 8,569 & 50.8 & 25.7 & 4,372 & 35.0 & 13.9 & 12 \\
\hline \multirow[t]{2}{*}{ Sex } & Male & 23.1 & 9.6 & 10,021 & 41.0 & 28.0 & 4,724 & 28.0 & 14.6 & 14 \\
\hline & Female & 30.5 & 9.2 & 10,667 & 51.9 & 24.7 & 5,969 & 37.1 & 13.9 & 16 \\
\hline \multirow{3}{*}{$\begin{array}{l}\text { Marital } \\
\text { status }\end{array}$} & Currently married & 25.2 & 10.1 & 13,068 & 44.8 & 27.6 & 6,294 & 30.6 & 15.0 & 19, \\
\hline & never married & 15.4 & 2.5 & 141 & 39.4 & 14.5 & 87 & 23.3 & 6.5 & 22 \\
\hline & divorced/separated/deserted & 30.1 & 8.2 & 7,479 & 50.6 & 24.2 & 4,312 & 36.5 & 13.2 & 11 \\
\hline \multirow[t]{5}{*}{ Education } & No Education & 24.5 & 6.9 & 13,633 & 38.9 & 14.5 & 3,693 & 27.0 & 8.2 & 17 \\
\hline & Below primary & 30.7 & 11.0 & 2,324 & 45.2 & 24.5 & 1,292 & 35.1 & 15.1 & 3,6 \\
\hline & Primary & 30.7 & 13.9 & 2,065 & 51.5 & 29.7 & 1,527 & 38.4 & 19.8 & 3,5 \\
\hline & Secondary & 33.0 & 16.7 & 2,341 & 53.7 & 36.7 & 3,093 & 43.6 & 27.0 & 5,1 \\
\hline & Higher & 33.6 & 19.7 & 323 & 51.8 & 32.6 & 1,088 & 46.9 & 29.1 & 1,2 \\
\hline \multirow[t]{3}{*}{ Religion } & Hindu & 25.8 & 9.2 & 17,281 & 46.6 & 26.4 & 8,572 & 31.7 & 14.1 & 25 \\
\hline & Muslim & 29.9 & 7.8 & 2,017 & 49.1 & 22.6 & 1,478 & 37.0 & 13.3 & 3,4 \\
\hline & Others & 36.0 & 13.4 & 1,390 & 49.0 & 31.0 & 644 & 39.5 & 18.1 & 2,0 \\
\hline \multirow[t]{2}{*}{ Wealth } & Poor & 21.6 & 7.0 & 9,033 & 41.6 & 17.8 & 4,632 & 27.4 & 10.1 & 13 \\
\hline & Non-poor & 31.1 & 11.2 & 11,655 & 51.3 & 32.5 & 6,061 & 37.0 & 17.5 & 17 \\
\hline \multirow{2}{*}{$\begin{array}{l}\text { Currently } \\
\text { working }\end{array}$} & Yes & 18.1 & 6.4 & 7,156 & 34.4 & 19.5 & 2,169 & 21.2 & 8.8 & 9,5 \\
\hline & No & 30.4 & 11.3 & 8,553 & 44.9 & 26.2 & 4,732 & 34.7 & 15.7 & 13 \\
\hline \multirow{2}{*}{$\begin{array}{l}\text { Ever used } \\
\text { smokeless } \\
\text { tobacco }\end{array}$} & Yes & 22.4 & 7.4 & 9,358 & 40.6 & 19.3 & 2,894 & 26.0 & 9.7 & 12 \\
\hline & No & 30.7 & 11.0 & 11,231 & 49.4 & 28.7 & 7,709 & 37.3 & 17.3 & 18 \\
\hline \multirow{2}{*}{$\begin{array}{l}\text { Ever } \\
\text { consumed } \\
\text { alcohol }\end{array}$} & Yes & 21.3 & 8.1 & 3,255 & 37.7 & 25.1 & 1,233 & 25.1 & 12.0 & 4,5 \\
\hline & No & 28.0 & 9.6 & 17,338 & 48.2 & 26.3 & 9,372 & 34.1 & 14.6 & 26 \\
\hline Total & & 26.9 & 9.4 & 20,589 & 47.0 & 26.1 & 10,603 & 32.8 & 14.2 & 31 , \\
\hline
\end{tabular}


Table 2

Estimates of odds ratio for hypertension and diabetes among elderly by their sociodemographic characterises in India

\begin{tabular}{|c|c|c|c|c|c|c|c|c|c|}
\hline \multirow[b]{3}{*}{ Age group } & & \multicolumn{4}{|l|}{ Rural } & \multicolumn{4}{|l|}{ Urban } \\
\hline & & \multicolumn{2}{|c|}{ Hypertension } & \multicolumn{2}{|l|}{ Diabetes } & \multicolumn{2}{|c|}{ Hypertension } & \multicolumn{2}{|l|}{ Diabetes } \\
\hline & & $\begin{array}{l}\text { Odds } \\
\text { ratio }\end{array}$ & $\begin{array}{l}\mathrm{Cl} \text { at } \\
95 \%\end{array}$ & $\begin{array}{l}\text { Odds } \\
\text { ratio }\end{array}$ & $\begin{array}{l}\mathrm{Cl} \text { at } \\
95 \%\end{array}$ & $\begin{array}{l}\text { Odds } \\
\text { ratio }\end{array}$ & $\begin{array}{l}\mathrm{Cl} \text { at } \\
95 \%\end{array}$ & $\begin{array}{l}\text { Odds } \\
\text { ratio }\end{array}$ & $\begin{array}{l}\mathrm{Cl} \text { at } \\
95 \%\end{array}$ \\
\hline & $60-69 \circledast$ & & & & & & & & \\
\hline & $70+$ & 1.02 & $\begin{array}{l}0.90- \\
1.15\end{array}$ & 0.91 & $\begin{array}{l}0.76- \\
1.08\end{array}$ & 1.19 & $\begin{array}{l}0.96- \\
1.46\end{array}$ & 0.93 & $\begin{array}{l}0.73- \\
1.17\end{array}$ \\
\hline \multirow[t]{2}{*}{ Sex } & Male ${ }^{\circledR}$ & & & & & & & & \\
\hline & Female & $1.47 \star \star \star$ & $\begin{array}{l}1.27- \\
1.69\end{array}$ & 1.15 & $\begin{array}{l}0.91- \\
1.43\end{array}$ & $1.43^{\star *}$ & $\begin{array}{l}1.09- \\
1.88\end{array}$ & $0.60 * \star \star$ & $\begin{array}{l}0.46- \\
0.80\end{array}$ \\
\hline \multirow[t]{3}{*}{ Marital status } & Currently married $\AA$ & & & & & & & & \\
\hline & never married & $0.45^{\star \star}$ & $\begin{array}{l}0.21- \\
0.95\end{array}$ & 0.20 ** & $\begin{array}{l}0.06- \\
0.65\end{array}$ & 0.88 & $\begin{array}{l}0.44- \\
1.77\end{array}$ & 0.59 & $\begin{array}{l}0.27- \\
1.25\end{array}$ \\
\hline & divorced/separated/deserted & 1.10 & $\begin{array}{l}0.96- \\
1.25\end{array}$ & 0.89 & $\begin{array}{l}0.72- \\
1.08\end{array}$ & 0.95 & $\begin{array}{l}0.73- \\
1.22\end{array}$ & $0.72^{\star \star}$ & $\begin{array}{l}0.55- \\
0.94\end{array}$ \\
\hline \multirow[t]{5}{*}{ Education } & No Education ${ }^{\circledR}$ & & & & & & & & \\
\hline & Below primary & $1.79 * \star \star$ & $\begin{array}{l}1.46- \\
2.20\end{array}$ & $1.98^{\star \star \star}$ & $\begin{array}{l}1.52- \\
2.59\end{array}$ & 1.31 & $\begin{array}{l}0.96- \\
1.79\end{array}$ & $1.60 * *$ & $\begin{array}{l}1.13^{-} \\
2.27\end{array}$ \\
\hline & Primary & $1.72^{\star \star \star}$ & $\begin{array}{l}1.43- \\
2.08\end{array}$ & $2.50 * \star \star$ & $\begin{array}{l}1.90- \\
3.30\end{array}$ & $1.77^{\star \star \star}$ & $\begin{array}{l}1.26- \\
2.48\end{array}$ & $2.34^{\star \star \star}$ & $\begin{array}{l}1.60- \\
3.42\end{array}$ \\
\hline & Secondary & $2.18^{\star \star \star}$ & $\begin{array}{l}1.84- \\
2.59\end{array}$ & $3.04^{\star \star \star}$ & $\begin{array}{l}2.40- \\
3.84\end{array}$ & $1.93^{\star \star \star}$ & $\begin{array}{l}1.43- \\
2.61\end{array}$ & $2.05^{\star \star \star}$ & $\begin{array}{l}1.51- \\
2.79\end{array}$ \\
\hline & Higher & $2.09 * \star \star$ & $\begin{array}{l}1.50- \\
2.91\end{array}$ & 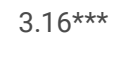 & $\begin{array}{l}2.01- \\
4.96\end{array}$ & $2.12^{\star \star \star}$ & $\begin{array}{l}1.40- \\
3.21\end{array}$ & $1.89 * *$ & $\begin{array}{l}1.20- \\
2.97\end{array}$ \\
\hline \multirow[t]{3}{*}{ Religion } & Hindu ${ }^{\circledR}$ & & & & & & & & \\
\hline & Muslim & 1.21 & $\begin{array}{l}0.99- \\
1.47\end{array}$ & 0.85 & $\begin{array}{l}0.64- \\
1.12\end{array}$ & 1.27 & $\begin{array}{l}0.98- \\
1.62\end{array}$ & 1.06 & $\begin{array}{l}0.79- \\
1.41\end{array}$ \\
\hline & Others & 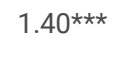 & $\begin{array}{l}1.16- \\
1.71\end{array}$ & $1.35^{\star \star}$ & $\begin{array}{l}1.01- \\
1.82\end{array}$ & 1.04 & $\begin{array}{l}0.76- \\
1.40\end{array}$ & $1.46^{\star \star}$ & $\begin{array}{l}1.07- \\
1.99\end{array}$ \\
\hline \multirow[t]{2}{*}{ Wealth } & Poor $\AA$ & & & & & & & & \\
\hline & Non-poor & $1.54^{\star \star \star}$ & $\begin{array}{l}1.37- \\
1.73\end{array}$ & 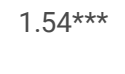 & $\begin{array}{l}1.30- \\
1.83\end{array}$ & 1.08 & $\begin{array}{l}0.87- \\
1.31\end{array}$ & $1.53^{\star \star \star}$ & $\begin{array}{l}1.23- \\
1.89\end{array}$ \\
\hline \multirow[t]{2}{*}{ Currently working } & Yes ${ }^{\circledR}$ & & & & & & & & \\
\hline & No & $1.89 * \star \star$ & $\begin{array}{l}1.67- \\
2.14\end{array}$ & 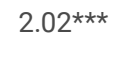 & $\begin{array}{l}1.68- \\
2.42\end{array}$ & $1.40 * \star \star$ & $\begin{array}{l}1.10- \\
1.77\end{array}$ & $1.52^{\star \star \star}$ & $\begin{array}{l}1.14- \\
2.01\end{array}$ \\
\hline \multirow{2}{*}{$\begin{array}{l}\text { Ever used smokeless } \\
\text { tobacco }\end{array}$} & Yes ${ }^{\circledR}$ & & & & & & & & \\
\hline & No & $1.28^{\star \star \star}$ & $\begin{array}{l}1.13- \\
1.44\end{array}$ & $1.45^{\star \star \star}$ & $\begin{array}{l}1.22- \\
1.73\end{array}$ & 1.08 & $\begin{array}{l}0.85- \\
1.36\end{array}$ & $1.66^{\star \star \star}$ & $\begin{array}{l}1.32- \\
2.09\end{array}$ \\
\hline \multirow[t]{2}{*}{ Ever consumed alcohol } & Yes ${ }^{\circledR}$ & & & & & & & & \\
\hline & No & 0.99 & $\begin{array}{l}0.85- \\
1.15\end{array}$ & 1.04 & $\begin{array}{l}0.83- \\
1.30\end{array}$ & 1.05 & $\begin{array}{l}0.81- \\
1.34\end{array}$ & 0.95 & $\begin{array}{l}0.72- \\
1.24\end{array}$ \\
\hline
\end{tabular}

Table 3 examined treatment-seeking for hypertension and diabetes among the elderly by socio-demographic characteristics. A higher percentage of older females were seeking treatment for hypertension and diabetes than their male counterparts. Furthermore, the result found that as education increases, the percentage of older people seeking treatment for hypertension also increases. Nearly 73 percent of non-poor sought treatment for hypertension, whereas almost 80 percent of non-poor people sought treatment for hypertension. Similarly, around 77 percent of poor elderly sought treatment for diabetes, whereas around 84 percent of non-poor elderly sought treatment for diabetes. 
Table 3

Treatment seeking for hypertension and diabetes among elderly by sociodemographic characterises

\begin{tabular}{|c|c|c|c|c|c|}
\hline & & Treatment seeking for hypertension & $\mathbf{N}$ & Treatment seeking for diabetes & $\mathbf{N}$ \\
\hline \multirow[t]{2}{*}{ Age group } & $60-69$ & 75.7 & 6,144 & 81.7 & 2,902 \\
\hline & $70+$ & 79.5 & 4,847 & 81.4 & 1,956 \\
\hline \multirow[t]{2}{*}{ Sex } & Male & 76.0 & 4,420 & 78.1 & 2,349 \\
\hline & Female & 78.4 & 6,571 & 84.9 & 2,509 \\
\hline \multirow[t]{3}{*}{ Marital status } & Currently married & 76.4 & 6,358 & 81.2 & 3,161 \\
\hline & never married & 73.2 & 56 & 62.7 & 15 \\
\hline & divorced/separated/deserted & 78.9 & 4,577 & 82.6 & 1,682 \\
\hline \multirow[t]{5}{*}{ Education } & No Education & 71.4 & 5,148 & 76.8 & 1,595 \\
\hline & Below primary & 78.8 & 1,354 & 78.3 & 593 \\
\hline & Primary & 81.1 & 1,446 & 86.8 & 757 \\
\hline & Secondary & 84.9 & 2,403 & 86.7 & 1,510 \\
\hline & Higher & 86.5 & 640 & 76.8 & 404 \\
\hline \multirow[t]{3}{*}{ Religion } & Hindu & 76.9 & 8,771 & 81.2 & 3,961 \\
\hline & Muslim & 78.4 & 1,358 & 82.5 & 495 \\
\hline & Others & 80.8 & 862 & 84.2 & 402 \\
\hline \multirow[t]{2}{*}{ Wealth } & Poor & 72.6 & 3,996 & 77.0 & 1,503 \\
\hline & Non-poor & 80.2 & 6,995 & 83.7 & 3,355 \\
\hline \multirow[t]{2}{*}{ Currently working } & Yes & 67.4 & 2,192 & 72.7 & 938 \\
\hline & No & 78.6 & 4,941 & 80.5 & 2,299 \\
\hline \multirow[t]{2}{*}{ Ever used smokeless tobacco } & Yes & 71.7 & 3,482 & 76.6 & 1,323 \\
\hline & No & 80.0 & 7,441 & 83.6 & 3,501 \\
\hline \multirow[t]{2}{*}{ Ever consumed alcohol } & Yes & 70.5 & 1,219 & 77.0 & 593 \\
\hline & No & 78.2 & 9,710 & 82.4 & 4,236 \\
\hline
\end{tabular}


Table 4

Logistic regression of treatment seeking for hypertension and diabetes among elderly by sociodemographic characterises

\begin{tabular}{|c|c|c|c|c|c|c|c|c|c|}
\hline & & \multicolumn{4}{|l|}{ Rural } & \multicolumn{4}{|l|}{ Urban } \\
\hline & & \multicolumn{2}{|c|}{ Hypertension } & \multicolumn{2}{|l|}{ Diabetes } & \multicolumn{2}{|c|}{ Hypertension } & \multicolumn{2}{|c|}{ Diabetes } \\
\hline & & $\begin{array}{l}\text { Odds } \\
\text { ratio }\end{array}$ & $\underset{\%}{\mathrm{Cl}}$ at 95 & $\begin{array}{l}\text { Odds } \\
\text { ratio }\end{array}$ & $\begin{array}{l}\mathrm{Cl} \text { at } 95 \\
\%\end{array}$ & Odds ratio & $\underset{\%}{\mathrm{Cl}}$ at 95 & $\begin{array}{l}\text { Odds } \\
\text { ratio }\end{array}$ & $\underset{\%}{\mathrm{Cl}}$ at 95 \\
\hline \multirow[t]{2}{*}{ Age group } & $60-69 \AA$ & & & & & & & & \\
\hline & $70+$ & 1.19 & $\begin{array}{l}0.96- \\
1.47\end{array}$ & 1.01 & $\begin{array}{l}0.70- \\
1.43\end{array}$ & 0.8989432 & $\begin{array}{l}0.62- \\
1.29\end{array}$ & 0.87 & $\begin{array}{l}0.54- \\
1.37\end{array}$ \\
\hline \multirow[t]{2}{*}{ Sex } & Male $\circledast$ & & & & & & & & \\
\hline & Female & 1.03 & $\begin{array}{l}0.78- \\
1.34\end{array}$ & 1.46 & $\begin{array}{l}0.89- \\
2.38\end{array}$ & 1.351669 & $\begin{array}{l}0.82- \\
2.20\end{array}$ & 1.38 & $\begin{array}{l}0.75- \\
2.53\end{array}$ \\
\hline \multirow[t]{3}{*}{ Marital status } & Currently married $®$ & & & & & & & & \\
\hline & never married & 0.27 & $\begin{array}{l}0.07- \\
1.00\end{array}$ & $0.05^{\star \star}$ & $\begin{array}{l}0.00- \\
0.50\end{array}$ & 2.244054 & $\begin{array}{l}0.35- \\
14.37\end{array}$ & 0.67 & $\begin{array}{l}0.09- \\
4.54\end{array}$ \\
\hline & divorced/separated/deserted & 0.98 & $\begin{array}{l}0.75- \\
1.25\end{array}$ & $0.50 * * *$ & $\begin{array}{l}0.32- \\
0.77\end{array}$ & 0.8387334 & $\begin{array}{l}0.52- \\
1.35\end{array}$ & 1.14 & $\begin{array}{l}0.65- \\
1.98\end{array}$ \\
\hline \multirow[t]{5}{*}{ Education } & No Education ${ }^{\circledR}$ & & & & & & & & \\
\hline & Below primary & 1.23 & $\begin{array}{l}0.86- \\
1.74\end{array}$ & 1.16 & $\begin{array}{l}0.68- \\
1.96\end{array}$ & 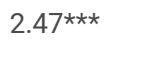 & $\begin{array}{l}1.37- \\
4.47\end{array}$ & 1.00 & $\begin{array}{l}0.49- \\
2.01\end{array}$ \\
\hline & Primary & 1.38 & $\begin{array}{l}0.99- \\
1.91\end{array}$ & $2.06^{\star \star}$ & $\begin{array}{l}1.15- \\
3.68\end{array}$ & $2.03^{\star \star}$ & $\begin{array}{l}1.68- \\
3.54\end{array}$ & $2.61^{\star *}$ & $\begin{array}{l}1.09- \\
6.25\end{array}$ \\
\hline & Secondary & 1.34 & $\begin{array}{l}0.98- \\
1.83\end{array}$ & 1.45 & $\begin{array}{l}0.90- \\
2.32\end{array}$ & $2.25^{\star \star \star}$ & $\begin{array}{l}1.31- \\
3.88\end{array}$ & 1.77 & $\begin{array}{l}0.91- \\
3.43\end{array}$ \\
\hline & Higher & 1.38 & $\begin{array}{l}0.77- \\
2.45\end{array}$ & 1.90 & $\begin{array}{l}0.75- \\
4.75\end{array}$ & $2.88^{\star \star \star}$ & $\begin{array}{l}1.40- \\
5.90\end{array}$ & 0.81 & $\begin{array}{l}0.32- \\
2.05\end{array}$ \\
\hline \multirow[t]{3}{*}{ Religion } & Hindu $\circledast$ & & & & & & & & \\
\hline & Muslim & 0.75 & $\begin{array}{l}0.54- \\
1.01\end{array}$ & 1.67 & $\begin{array}{l}0.89- \\
3.10\end{array}$ & $1.78^{\star \star}$ & $\begin{array}{l}1.10- \\
2.86\end{array}$ & 1.37 & $\begin{array}{l}0.61- \\
3.05\end{array}$ \\
\hline & Others & 1.10 & $\begin{array}{l}0.77- \\
1.54\end{array}$ & 1.26 & $\begin{array}{l}0.72- \\
2.19\end{array}$ & $3.05^{\star \star \star}$ & $\begin{array}{l}1.63- \\
5.72\end{array}$ & 1.29 & $\begin{array}{l}0.62- \\
2.66\end{array}$ \\
\hline \multirow[t]{2}{*}{ Wealth } & Poor® & & & & & & & & \\
\hline & Non-poor & $1.52^{\star \star \star}$ & $\begin{array}{l}1.23- \\
1.88\end{array}$ & $1.80 * * \star$ & $\begin{array}{l}1.27- \\
2.56\end{array}$ & $1.74^{\star \star \star}$ & $\begin{array}{l}1.18- \\
2.56\end{array}$ & 1.14 & $\begin{array}{l}0.70- \\
1.82\end{array}$ \\
\hline \multirow[t]{2}{*}{ Currently working } & Yes $®$ & & & & & & & & \\
\hline & No & $1.68^{\star \star \star}$ & $\begin{array}{l}1.35- \\
2.08\end{array}$ & $1.45^{\star \star}$ & $\begin{array}{l}1.01- \\
2.09\end{array}$ & $1.63^{\star \star}$ & $\begin{array}{l}1.10- \\
2.42\end{array}$ & 1.72 & $\begin{array}{l}0.80- \\
3.70\end{array}$ \\
\hline \multirow{2}{*}{$\begin{array}{l}\text { Ever used smokeless } \\
\text { tobacco }\end{array}$} & Yes $®$ & & & & & & & & \\
\hline & No & $1.17 \star \star \star$ & $\begin{array}{l}0.94- \\
1.47\end{array}$ & 1.34 & $\begin{array}{l}0.92- \\
1.94\end{array}$ & 1.450114 & $\begin{array}{l}0.98- \\
2.14\end{array}$ & 1.07 & $\begin{array}{l}0.64- \\
1.74\end{array}$ \\
\hline \multirow[t]{2}{*}{ Ever consumed alcohol } & Yes $®$ & & & & & & & & \\
\hline & No & $1.30 \star \star$ & $\begin{array}{l}1.00- \\
1.69\end{array}$ & 1.28 & $\begin{array}{l}0.83- \\
1.97\end{array}$ & 1.500037 & $\begin{array}{l}0.95- \\
2.36\end{array}$ & 0.76 & $\begin{array}{l}0.43- \\
1.32\end{array}$ \\
\hline
\end{tabular}

Table 4 presents estimates for odds ratio examining the treatment-seeking for hypertension and diabetes among the elderly, separately for rural and urban areas. Results found that older people with higher education were 2.88 times $(\mathrm{OR}=2.88 ; \mathrm{C} . \mathrm{I}=1.40-5.90)$ more likely to sought treatment for hypertension than uneducated older people in urban areas. As expected, results noticed a higher treatment seeking among non-poor elderly than their counterparts. It was noticed that non-poor older people were more likely to sought treatment for hypertension $(\mathrm{OR}=1.52 ; \mathrm{C} . \mathrm{I} .=1.23-1.88)$ and diabetes $(\mathrm{OR}=1.45 ; \mathrm{C} . \mathrm{I} .=1.01-2.09)$ than older people who were poor in rural areas. Similarly, non-poor older people in urban areas were 1.74 times $(O R=1.74 ; C . I$. $=1.18-2.56)$ more likely to sought treatment for hypertension than their counterparts.

Table 5 depicts the urban-rural gap in the prevalence of hypertension among the elderly with the help of Fairlie's decomposition analysis. The negative sign to the coefficient depicts that the particular variable contributes to narrowing the inequality gap of hypertension between rural-urban and vice-versa. Results found that age, sex, and wealth are the three variables that narrow the inequality in the rural-urban prevalence of hypertension. In simpler terms, it means that age, sex, and wealth are the variables that are reducing the gap in the prevalence of rural-urban hypertension among the elderly. Furthermore, the result noticed that education status alone accounts for more than four-fifths (88.62\%) of the inequality in the prevalence of urban-rural hypertension. It means that the 
urban-rural differential in the prevalence of hypertension could be attributed to the educational status of the elderly. The current working status of the elderly is another factor that determines the inequality in the prevalence of hypertension between urban and rural areas.

Table 5

Decomposition results of the urban and rural gap in hypertension among elderly in India

\begin{tabular}{|lllllll|}
\hline & Coefficient & Standard error & P-value & Lower Cl at 95\% & Upper Cl at 95\% & Percent contribution \\
\hline Age & -0.0010233 & 0.000705 & 0.147 & -0.0024 & 0.000358 & -1.93 \\
\hline Sex & -0.0063831 & 0.002683 & 0.017 & -0.01164 & -0.00113 & -12.03 \\
\hline Marital Status & 0.0003314 & 0.000918 & 0.718 & -0.00147 & 0.002131 & 0.62 \\
\hline Education & 0.0470134 & 0.010132 & 0 & 0.027155 & 0.066872 & 88.62 \\
\hline Religion & 0.0005718 & 0.0005 & 0.252 & -0.00041 & 0.001551 & 1.08 \\
\hline Wealth & -0.0000606 & 0.000224 & 0.787 & -0.0005 & 0.000378 & -0.11 \\
\hline Currently working & 0.0106343 & 0.003804 & 0.005 & 0.003179 & 0.01809 & 20.05 \\
\hline Ever used smokeless tobacco & 0.0018305 & 0.00391 & 0.64 & -0.00583 & 0.009495 & 3.45 \\
\hline Ever consumed alcohol & 0.0002218 & 0.000435 & 0.61 & -0.00063 & 0.001074 & 0.42 \\
\hline Total Explained = 0.05305 & & & & & & \\
\hline
\end{tabular}

Table 6 depicts the urban-rural gap in the prevalence of diabetes among the elderly with the help of Fairlie's decomposition analysis. Education status of the elderly accounts for more than half (52.02\%) of the inequality in the rural-urban prevalence of diabetes. Other important factors that are contributing to the inequality in the rural-urban prevalence of diabetes are ever-used smokeless tobacco (19.54\%), currently working status (15.58\%), and sex (9.83\%).

Table 6

Decomposition results of the urban and rural gap in diabetes among elderly in India

\begin{tabular}{|lllllll|}
\hline & Coefficient & Standard error & P-value & Lower Cl at 95\% & Upper Cl at 95\% & Percent contribution \\
\hline Age & 0.000193 & 0.000502 & 0.7 & -0.00079 & 0.001177 & 0.32 \\
\hline Sex & 0.005856 & 0.001917 & 0.002 & 0.002098 & 0.009614 & 9.83 \\
\hline Marital Status & 0.001204 & 0.000786 & 0.126 & -0.00034 & 0.002744 & 2.02 \\
\hline Education & 0.030991 & 0.007798 & 0 & 0.015707 & 0.046274 & 52.02 \\
\hline Religion & 0.000448 & 0.00031 & 0.149 & -0.00016 & 0.001055 & 0.75 \\
\hline Wealth & 0.00026 & 0.000675 & 0.7 & -0.00106 & 0.001584 & 0.44 \\
\hline Currently working & 0.009285 & 0.003141 & 0.003 & 0.003128 & 0.015441 & 15.58 \\
\hline Ever used smokeless tobacco & 0.011639 & 0.003046 & 0 & 0.005669 & 0.017608 & 19.54 \\
\hline Ever consumed alcohol & -0.00033 & 0.000666 & 0.62 & -0.00163 & 0.000975 & -0.55 \\
\hline Total Explained = 0.059576 & & & & & & \\
\hline
\end{tabular}

\section{Discussion}

India is currently living through a prodigious wave of demographic change where an increase in life expectancy and decline in fertility have resulted in a dramatic rise in the population of elderly aged 60 years and above. Accompanying the issue of population ageing is the increasing prevalence of chronic diseases among the elderly. The demographic vis-à-vis the epidemiological transition in India has shifted a significant share of disease burden to the older population. Therefore, the objective of this study was to examine the rural-urban differentials in the prevalence and associated factors of chronic diseases (hypertension and diabetes) among the elderly. Also, the study aimed to examine the treatment-seeking approach of the elderly concerning the said diseases.

\subsection{Prevalence and factors associated with hypertension and diabetes}

The results explicitly found that the prevalence of hypertension and diabetes was much higher among the elderly in urban areas than in rural areas. Rapid urbanization is directing a change in lifestyle accompanied by inadequate physical activity and over-dependence on junk food among elderly in urban areas, which is the prime cause of higher diabetes among elderly in urban areas [13-16]. Previous studies are in concordance with this study in finding a higher prevalence of hypertension in urban areas than in rural areas $[6,17]$. The higher prevalence of hypertension in urban areas may have arisen due to the rise in cardiovascular disease risk factors among the urban population [18]. Unhealthy dietary practices, consumption of tobacco, and sedentary habits occurring due to rapid urbanization have also contributed to the growing epidemic of hypertension in urban areas of the country [6]. Furthermore, the differences in hypertension prevalence between urban and rural areas could be explained by the differences in socio-economic conditions, risk factors, and quality of healthcare services provided in rural and urban areas [6]. 
However, previous studies noted a higher risk of hypertension among the elderly with an increase in age [19, 20] ; this study failed to notice the statistical differences between a high risk of hypertension and an increase in age among elderly. In rural and urban areas, females were more likely to be diagnosed with hypertension than their male counterparts. Previous studies also noticed this difference and concluded that older females are at higher risk of developing hypertension than older males [20]. It may be due to insufficiency of ovarian hormones during the postmenopausal period [20]. Studies have advocated that ovarian hormones, especially estrogen, may have the potentials to keep blood pressure lower in premenopausal women, and lack of estrogen may be held accountable for elevated blood pressure in postmenopausal women, thus rising hypertension among them [21]. Furthermore, women at advanced age become socially more vulnerable to hypertension as most of such women do not have their spouse alive, affecting their healthcare-seeking and treatment [20]. Female elderly in urban areas were at lower risk of diabetes than their male counterparts in urban areas. Few studies are in concordant with this finding [22, 23]. However, few previous studies noted a higher prevalence of diabetes among female elderly than males [24, 25]. The higher prevalence of diabetes among older men than in older women could be associated with a large amount of visceral fat in men [26].

Among other factors, a higher level of education was responsible for the increased odds of hypertension and diabetes. Educated elderly were at higher risk of having hypertension and diabetes in rural as well as in urban areas than uneducated elderly. The results from decomposition analysis further clarified that education contributes to more than one-fourth of the inequality in the prevalence of hypertension and diabetes among the elderly in India. Previous studies also noticed that the risk of hypertension was higher among the educated elderly [20,27]. Education levels might improve wealth status, make people follow a sedentary lifestyle, and eventually increase the odds of hypertension [28]. The study found that the diagnosis of hypertension and diabetes was higher among non-poor than in poor. Previous studies have also highlighted a higher burden of diabetes and hypertension among people from the richest wealth quintiles $[17,29-32]$. Although the odds of hypertension were higher among non-poor than in poor, the prevalence of hypertension among the elderly suggests that hypertension is also quite prevalent among the poor. Rightly, it was stated that hypertension is not the chronic condition of only the affluent, and it may occur to the poor as well [33]. In the rural area, the odds of diagnosis of hypertension showed a marked positive gradient from poor to non-poor household. It was odds of diabetes that were higher among the elderly in non-poor households than those elderly who belonged to poor households, both in the urban and rural areas. Non-poor elderly tend to adopt a sedentary lifestyle leading to an increased risk of diabetes among them.

\subsection{Prevalence and factors associated with treatment-seeking of hypertension and diabetes}

The most important factor in the discourse of treatment-seeking of hypertension among the elderly is the household wealth status. Results found that the elderly from non-poor households had higher odds to sought treatment for hypertension in rural as well as in urban areas. Previous studies attributed household wealth as an indicator of high treatment-seeking for hypertension in India and elsewhere [34, 35]. It is expected that the elderly from affluent households would seek higher treatment for hypertension than their poor counterparts because of their financial stability. Similarly, the odds of treatmentseeking for diabetes were higher among non-poor rural elderly than their counterparts in poor households. The high cost of treatment could be a cause for lesser odds of seeking treatment for diabetes among the elderly in poor households. Studies have reported that the high cost of treatment could be a deterring factor in utilizing the treatment for hypertension and diabetes [36]. Furthermore, in India, healthcare is financed by out-of-pocket payments and private Health care insurance, which may further favour people from higher socio-economic groups leaving poor people behind [37]. The health financing system in India is out rightly complex than other developing countries [38], and a large chunk of the country's healthcare is provided by unregulated expensive private sector favouring the rich [39-41].

The results noted that the odds of treatment-seeking for hypertension and diabetes were higher among non-working elderly than working elderly. This may be a paragon of paradoxes as common thought reaches the conclusion that working elderly should have better treatment-seeking than their non-working counterparts; however, it is vice-versa. Why it is, so that non-working elderly have higher odds of treatment-seeking than their counterparts. Let us understand this contradiction- Economic dependency is higher among the elderly [42], and $60+$ age category is generally a category of the retired working-class, and whosoever is working even after their 60 s might require money as they might be poor and may never had permanent income in their entire life. This could be the reason for lower treatment-seeking among working elderly. The odds of treatment-seeking for hypertension were higher among educated elderly than uneducated elderly in urban areas. It is understood that educated elderly might have a better financial status which may further aggravate treatment-seeking. Furthermore, a previous study in the Indian context noted that the elderly with higher education levels are more likely to go for treatment than the uneducated elderly [43].

\section{Strengths And Limitations Of The Study}

The study has some potential limitations. Technically, LASI is a longitudinal survey; however, we were unable to present any trends because this was the first wave of released data. The study used cross-sectional data (since this was the first wave) and, therefore, causality to be inference carefully. Another potential limitation is that the survey did not ask the question on type of treatment for hypertension and diabetes that could differentiate whether the person was taking medicine, following a weight loss program, or observing a change in eating habits. Despite the limitations mentioned above, this study has some considerable strengths. First, hypertension and diabetes are not self-reported and were diagnosed by doctors/health professionals. Self-reported information may be affected by recall biases and reporting errors that may vary by socio-demographic characteristics [17]. Second, this is the latest data (released in 2021) that allow us to present the current picture of the health measures of the elderly by encompassing the sample selection from each state in the country.

\section{Conclusion}

Our study found that the prevalence of hypertension and diabetes was higher among the elderly in urban areas than in rural areas. Hypertension was more prevalent among elderly females in urban areas, highly-educated elderly, non-poor rural elderly, and elderly who were currently not working. Similarly, the risk of diabetes was more prevalent among educated elderly, non-poor elderly, and elderly currently not working. Furthermore, the treatment-seeking for hypertension

Page 9/12 
was higher among educated elderly in urban areas, non-poor elderly, and elderly not currently working. Similarly, the treatment-seeking for diabetes was higher among non-poor rural elderly and elderly not working in the rural area. The study has a base to provide some policy implications. The unification of geriatric healthcare with the general healthcare system at the primary healthcare level can be a decisive stride in ameliorating the overall health status of older people. The policy-makers should propitious their efforts to address the vulnerability of women in rural as well as in urban areas while designing hypertension prevention and control programs. Since treatment-seeking is relatively low among elderly in poor households, practices must be identified for a povertystricken elderly population to overcome the financial barriers that may prevent the elderly from seeking and complying with treatment.

\section{Declarations}

Ethics approval and consent to participate: The data is freely available on request and survey agencies that conducted the field survey for the data collection have collected a prior consent from the respondent. The data can be accessed from:

https://iipsindia.ac.in/sites/default/files/LASI_DataRequestForm_0.pdf

Consent for publication: Not applicable

Availability of data and materials: The study utilises secondary source of data which is available on request to everyone through https://iipsindia.ac.in/sites/default/files/LASI_DataRequestForm_0.pdf.

Competing Interest: The authors declare that they have no competing interests.

Funding: Authors did not received any funding to carry out this research.

Author's Contribution: The concept was drafted by SK and SC. SK contributed to the analysis design. SC advised on the paper and assisted in paper conceptualization. SC, RP, and SSG contributed in the comprehensive writing of the article. All authors read and approved the final manuscript.

Acknowledgements: Not applicable

\section{Abbreviations}

\section{Cl}

Confidence Interval

LASI

Longitudinal Ageing Study in India

NCD

Non-communicable Disease

OR

Odds Ratio

\section{References}

1. GBD 2015 Risk Factors Collaborators. Global, regional, and national comparative risk assessment of 79 behavioural, environmental and occupational, and metabolic risks or clusters of risks, 1990-2015: a systematic analysis for the Global Burden of Disease Study 2015. Lancet, 2016; 388(10053):16591724. https://doi.org/10.1016/S0140-6736(16)31679-8

2. Rapsomaniki E, Timmis A, George J, et al. Blood pressure and incidence oftwelve cardiovascular diseases: lifetime risks, healthy life-years lost, and agespecific associations in 25 million people.Lancet Lond Engl.2014;383(9932):1899-1911.

https://doi.org/10.1016/S0140-6736(14)60685-1.

3. Rodgers, A., Lawes, C., \& MacMahon, S. (2000). Reducing the global burden of blood pressure-related cardiovascular disease. Journal of hypertension. Supplement: official journal of the International Society of Hypertension, 18(1), S3-S6.

Available at: https://pubmed.ncbi.nlm.nih.gov/10939783/.

4. Porapakkham, Y \& Pattaraarchachai, Junya \& Aekplakorn, W. (2008). Prevalence, awareness, treatment and control of hypertension and diabetes mellitus among the elderly: The 2004 National Health Examination Survey III, Thailand. Singapore medical journal. 49. 868-73.

Available at: http://www.smj.org.sg/sites/default/files/4911/4911a2.pdf.

5. Joshi SR, Parikh RM. India-diabetes capital of the world: now heading towards hypertension. The Journal of the Association of Physicians of India. 2007 May;55:323-324. PMID: 17844690. Available at: http://www.japi.org/may2007/E-323.pdf.

6. Anchala, R., Kannuri, N. K., Pant, H., Khan, H., Franco, O. H., Di Angelantonio, E., \& Prabhakaran, D. (2014). Hypertension in India: a systematic review and meta-analysis of prevalence, awareness, and control of hypertension. Journal of hypertension, 32(6), 1170-1177.

https://doi.org/10.1097/HJH.0000000000000146.

7. Siddiqui S, Waghdhare S, Panda M, Sinha S, Singh P, Dubey S, Jha S. Regional prevalence of gestational diabetes mellitus in North India. J Diabetol 2019;10:25-8.

https://doi.org/4103/jod.jod_32_18. 
8. Viswanathan Mohan, Prashant Mathur, Raj Deepa, Mohan Deepa, D.K. Shukla, Geetha R. Menon, Krishnan Anand, Nimesh G. Desai, Prashant P. Joshi, J. Mahanta, K.R. Thankappan, Bela Shah,Urban rural differences in prevalence of self-reported diabetes in India-The WHO-ICMR Indian NCD risk factor surveillance,Diabetes Research and Clinical Practice,Volume 80, Issue 1,2008,Pages 159-168,ISSN 0168-8227,

https://doi.org/10.1016/j.diabres.2007.11.018.

9. Geldsetzer, P., Manne-Goehler, J., Theilmann, M., Davies, J. I., Awasthi, A., Vollmer, S., Jaacks, L. M., Bärnighausen, T., \& Atun, R. (2018). Diabetes and Hypertension in India: A Nationally Representative Study of 1.3 Million Adults. JAMA internal medicine, 178(3), 363-372.

https://doi.org/10.1001/jamainternmed.2017.8094

10. Chomi, E.N. , Mujinja, P.G.M. , Enemark, U. , Hansen, K. , Kiwara, A.D. Health care seeking behaviour and utilisation in a multiple health insurance system: does insurance affiliation matter? Int J Equity Health. 2014; 13: 25. https://doi.org/10.1186/1475-9276-13-25

11. Singh AK, Mani K, Krishnan A, Aggarwal P, Gupta SK. Prevalence, Awareness, Treatment and Control of Diabetes Among Elderly Persons in an Urban Slum of Delhi. Indian J Community Med 2012;37:236-9.

https://doi.org/4103/0970-0218.103472

12. Grueninger U. J. (1995). Arterial hypertension: lessons from patient education. Patient education and counseling, 26(1-3), 37-55. https://doi.org/10.1016/0738-3991(95)00750-t.

13. Ebrahim, S., Kinra, S., Bowen, L., Andersen, E., Ben-Shlomo, Y., Lyngdoh, T., \& Indian Migration Study group. (2010). The effect of rural-to-urban migration on obesity and diabetes in India: a cross-sectional study. PLoS Med, 7(4), e1000268. https://doi.org/10.1371/journal.pmed.1000268

14. Cantarero-Prieto, D., Pascual-Sáez, M., \& Blázquez-Fernández, C. (2018). Social isolation and multiple chronic diseases after age 50: a European macroregional analysis. PloS one, 13(10), e0205062. https://doi.org/10.1371/journal.pone.0205062

15. Srivastava, S., Anwar, T., Patel, R., \& Chauhan, S. (2020). Dynamics of chronic diseases in metro and non-metro regions of India: evidence from India Human Development Survey I and II. International Journal, 6(8), 322. http://dx.doi.org/10.18203/issn.2454-2156.IntJSciRep20203116

16. Chakraborty, S., \& Sengupta, A. (2020). An Epidemiological Study on Prameha (Diabetes Mellitus) in Kolkata. International Journal of Ayurveda and Pharma Research, 21-26.

17. Moser, K. A., Agrawal, S., Smith, G. D., \& Ebrahim, S. (2014). Socio-demographic inequalities in the prevalence, diagnosis and management of hypertension in India: analysis of nationally-representative survey data. PloS one, 9(1), e86043. https://doi.org/10.1371/journal.pone.0086043

18. Jeemon, P., \& Reddy, K. S. (2010). Social determinants of cardiovascular disease outcomes in Indians. The Indian journal of medical research, $132(5), 617$. https://doi.org/4103/0971-5916.73415

19. Swami, H. M., Bhatia, V., Gupta, M., Bhatia, S. P. S., \& Sood, A. (2002). Population based study of hypertension among the elderly in northern India. Public Health, 116(1), 45-49. https://doi.org/10.1038/sj.ph.1900819

20. Hanif, A. A. M., Shamim, A. A., Hossain, M. M., Hasan, M., Khan, M. S. A., Hossaine, M., \& Mridha, M. K. (2021). Gender-specific prevalence and associated factors of hypertension among elderly Bangladeshi people: findings from a nationally representative cross-sectional survey. BMJ open, 11(1), e038326. http://dx.doi.org/10.1136/bmjopen-2020-038326

21. Raghvendra, K., Dubey, S. O., Bruno, I., \& Edwin, K. J. (2002). Sex hormones and hypertension. Cardiovascular Research, 53(3), 688-708.

22. Tripathy, J. P., Thakur, J. S., Jeet, G., Chawla, S., Jain, S., Pal, A., \& Saran, R. (2017). Prevalence and risk factors of diabetes in a large community-based study in North India: results from a STEPS survey in Punjab, India. Diabetology \& metabolic syndrome, 9(1), 1-8. https://doi.org/10.1186/s13098-0170207-3

23. Kutty, V. R., Dilip, T. R., Archana, A. R., Gopinathan, S., \& Ramanathan, M. (2018). Shifting pattern of diabetes among the elderly in India: Evidence from the national sample survey organization's data, 2004-2014. International Journal of Noncommunicable Diseases, 3(2), 67.

https://doi.org/10.4103/jncd.jncd_37_17

24. Singh, A. K., Kalaivani Mani, A. K., Aggarwal, P., \& Gupta, S. K. (2012). Prevalence, awareness, treatment and control of diabetes among elderly persons in an urban slum of Delhi. Indian journal of community medicine: official publication of Indian Association of Preventive \& Social Medicine, $37(4), 236$. https://doi.org/4103/0970-0218.103472

25. Kapil, U., Khandelwal, R., Ramakrishnan, L., Khenduja, P., Gupta, A., Pandey, R. M., \& Belwal, R. S. (2018). Prevalence of hypertension, diabetes, and associated risk factors among geriatric population living in a high-altitude region of rural Uttarakhand, India. Journal of family medicine and primary care, 7(6), 1527. https://doi.org/4103/jfmpc.jfmpc_108_18

26. Nordstrom, A., Hadrévi, J., Olsson, T., Franks, P. W., \& Nordstrom, P. (2016). Higher prevalence of type 2 diabetes in men than in women is associated with differences in visceral fat mass. The Journal of Clinical Endocrinology \& Metabolism, 101(10), 3740-3746. https://doi.org/10.1210/jc.2016-1915

27. Al Kibria, G. M., Burrowes, V., Choudhury, A., Sharmeen, A., \& Swasey, K. (2019). Sex differences in prevalence and associated factors of prehypertension and hypertension among Bangladeshi adults. International Journal of Cardiology Hypertension, 1, 100006. https://doi.org/10.1016/j.ijchy.2019.100006

28. Yabiku, S. T., \& Schlabach, S. (2009). Social change and the relationships between education and employment. Population research and policy review, 28(4), 533-549. https://doi.org/10.1007/s11113-008-9117-2

29. Paulsen, M. S., Andersen, M., Munck, A. P., Larsen, P. V., Hansen, D. G., Jacobsen, I. A., \& Sondergaard, J. (2012). Socio-economic status influences blood pressure control despite equal access to care. Family practice, 29(5), 503-510. https://doi.org/10.1093/fampra/cmr130

30. Tareque, M. I., Koshio, A., Tiedt, A. D., \& Hasegawa, T. (2015). Are the rates of hypertension and diabetes higher in people from lower socioeconomic status in Bangladesh? Results from a nationally representative survey. PloS one, 10(5), e0127954. https://doi.org/10.1371/journal.pone.0127954

31. Psaltopoulou, T., Hatzis, G., Papageorgiou, N., Androulakis, E., Briasoulis, A., \& Tousoulis, D. (2017). Socioeconomic status and risk factors for cardiovascular disease: impact of dietary mediators. Hellenic journal of cardiology, 58(1), 32-42. https://doi.org/10.1016/j.hjc.2017.01.022

Page $11 / 12$ 
32. Lloyd-Sherlock, P., Beard, J., Minicuci, N., Ebrahim, S., \& Chatterji, S. (2014). Hypertension among older adults in low-and middle-income countries: prevalence, awareness and control. International journal of epidemiology, 43(1), 116-128. https://doi.org/10.1093/ije/dyt215

33. Olshansky, S. J., \& Ault, A. B. (1986). The fourth stage of the epidemiologic transition: the age of delayed degenerative diseases. The Milbank Quarterly, 355-391. https://doi.org/10.2307/3350025

34. Prenissl, J., Manne-Goehler, J., Jaacks, L. M., Prabhakaran, D., Awasthi, A., Bischops, A. C., \& Geldsetzer, P. (2019). Hypertension screening, awareness, treatment, and control in India: a nationally representative cross-sectional study among individuals aged 15 to 49 years. PLoS medicine, $16(5)$, e1002801. https://doi.org/10.1371/journal.pmed.1002801

35. Saju, M. D., Allagh, K. P., Scaria, L., Joseph, S., \& Thiyagarajan, J. A. (2020). Prevalence, awareness, treatment, and control of hypertension and its associated risk factors: results from baseline survey of SWADES family cohort study. International journal of hypertension, 2020. https://doi.org/10.1155/2020/4964835

36. Rao, C. R., Kamath, V. G., Shetty, A., \& Kamath, A. (2014). Treatment compliance among patients with hypertension and type 2 diabetes mellitus in a coastal population of Southern India. International journal of preventive medicine, 5(8), 992.

37. Kumar, K., Shukla, A., Singh, A., Ram, F., \& Kowal, P. (2016). Association between wealth and health among older adults in rural China and India. The Journal of the Economics of Ageing, 7, 43-52. https://doi.org/10.1016/j.jeoa.2016.02.002

38. Chatterjee, P. (2010). India tries to break cycle of health-care debt. World Health Organization. Bulletin of the World Health Organization, 88(7), 486. https://www.scielosp.org/pdf/bwho/2010.v88n7/486-487

39. Evans, J. M., Kiran, P. R., \& Bhattacharyya, O. K. (2011). Activating the knowledge-to-action cycle for geriatric care in India. Health Research Policy and Systems, 9(1), 1-10. https://doi.org/10.1186/1478-4505-9-42

40. Dey, S., Nambiar, D., Lakshmi, J. K., Sheikh, K., \& Reddy, K. S. (2012). Health of the elderly in India: challenges of access and affordability. In Aging in Asia: Findings from new and emerging data initiatives. National Academies Press (US).

41. Bloom, D. E., Cafiero, E. T., McGovern, M. E., Prettner, K., Stanciole, A., Weiss, J., \& Rosenberg, L. (2013). The economic impact of non-communicable disease in China and India: estimates, projections, and comparisons(No. w19335). National Bureau of Economic Research.

https://www.nber.org/system/files/working_papers/w19335/w19335.pdf

42. Kumar, S., \& Kumar, K. A. (2019). Living arrangement and economic dependency among the elderly in India: a comparative analysis of EAG and non EAG states. Ageing International, 44(4), 352-370. https://doi.org/10.1007/s12126-019-9344-3

43. Srivastava, S., \& Gill, A. (2020). Untreated morbidity and treatment-seeking behaviour among the elderly in India: Analysis based on National Sample Survey 2004 and 2014. SSM-population health, 10, 100557. https://doi.org/10.1016/j.ssmph.2020.100557 